\title{
Open-Heart Surgery for a Pregnant Woman With Infective Endocarditis After Cesarean
}

\author{
Fudong Fan ${ }^{\mathrm{a}}$, Qing Zhou ${ }^{\mathrm{a}}$, Jun Pan ${ }^{\mathrm{a}}$, Dongjin Wang ${ }^{\mathrm{a}, \mathrm{b}}$
}

\begin{abstract}
The presentation of infective endocarditis (IE) in pregnancy is mild, often with a life-threatening consequence. Usually the patient cannot be satisfactorily treated by antibiotics but needs surgery to remove the vegetation. We present a case of pregnant woman with IE who was successfully treated by antibiotics, cesarean and cardiothoracic surgery. In conclusion, IE in pregnancy can be cured by comprehensive treatments.
\end{abstract}

Keywords: Infective endocarditis; Pregnancy; Cardiothoracic surgery

\section{Introduction}

Infective endocarditis (IE) is typically limited to patients with preexisting valvular abnormalities or a history of intravenous drug use [1]. IE is a rare life-threatening complication of pregnancy and the incidence is between $0.006 \%$ and $0.0125 \%$ with the maternal and fetal mortality being $22.1-33 \%$ and $14.7-$ $29 \%$, respectively [2-4]. Here we report an unusual case of pregnancy complicated by Staphylococcus aureus IE with large vegetation at the bicuspid aortic valve. Although the neonates died from respiratory failure eventually, the mother was successfully cured after surgical operation.

\section{Case Report}

A 29-year-old woman with intrauterine pregnancy (33 weeks and 5 days) underwent 1 -week fever, chest tightness and asthma. In a local hospital, blood samples were collected and

Manuscript accepted for publication February 12, 2016

aDepartment of Cardiothoracic Surgery, The Affiliated Drum Tower Hospital of Nanjing University Medical School, China

${ }^{b}$ Corresponding Author: Dongjin Wang, Department of Cardiothoracic Surgery, The Affiliated Drum Tower Hospital of Nanjing University Medical School, 321 Zhongshan Road, Nanjing 210008, China.

Email: gldjw@163.com

doi: http://dx.doi.org/10.14740/jmc2434w
Staphylococcus aureus had been cultured. After treated by antibiotic therapy with vancomycin and piperacillin tazobactam for 4 days, the patient still had a fever of $39.7^{\circ} \mathrm{C}$ with oxygenation dysfunction on room air, so she was transferred to our hospital.

On admission, the physical examination revealed 1) a temperature of $39.7^{\circ} \mathrm{C}$ with no shaking chills, 2) respiration of 32 breaths $/ \mathrm{min}$, heart rate of $139 \mathrm{breaths} / \mathrm{min}$, blood pressure of $112 / 53 \mathrm{~mm} \mathrm{Hg}$ and oxygen saturation of $90 \%\left(\mathrm{FiO}_{2} 100 \%\right)$, 3) fine moist rale in both lower lung fields, and 4) a diastolic murmur in the aortic area and an apical pansystolic murmur. The laboratory examination revealed white blood cell count of $19,100 / \mathrm{mm}^{3}$ with $87.6 \%$ of neutrophils, and atrial natriuretic peptide of $1,280 \mathrm{pg} / \mathrm{mL}$. Transthoracic echocardiography demonstrated a large vegetation $(3.1 \times 1.5 \mathrm{~cm})$ attached at the bicuspid aortic valve leaflet (Fig. 1A), with mild to moderate aortic stenosis and moderate to severe aortic regurgitation, severe mitral regurgitation, moderate tricuspid regurgitation and dilations of the left atrium and left ventricle (Fig. 1B). The left ventricle ejection fraction was $56 \%$. Her initial diagnosis was IE, acute left heart failure, and pregnancy. Repeated blood was collected and cultured from three specimens taken $30 \mathrm{~min}$ apart. She was treated by antibiotics, diuretic and inotropic drugs.

On the second day after admission, the patient underwent cesarean and delivered a male infant with severe hypoxemia. Then the boy was given tracheal intubation and transferred to neonatal intensive care unit. Unfortunately, the boy died from respiratory failure 3 days later. After the cesarean, the patient was also treated by vancomycin and piperacillin tazobactam, but with consistent blood temperature of $39.6{ }^{\circ} \mathrm{C}$. The white blood count was $24,200 / \mathrm{mm}^{3}$ with $93.0 \%$ of neutrophils.

On the sixth day after admission, the patient was transferred to cardiothoracic surgery unit and underwent further operation. The intraoperative findings revealed bicuspid aortic valve, fusion of the commissure between the non-coronary and the right leaflet, several vegetations at the ventricular surface of the leaflets with a maximum size of $3.0 \times 2.0 \mathrm{~cm}$ and formation of an annular abscess. Myocardial tissues necrosis occurred under the ring of aortic valve. Severe mitral regurgitation and moderate tricuspid regurgitation were found. The operation involved removal of the vegetations and abscess, covering the defect with the autologous pericardium patch, aortic valve replacement with mechanical prosthesis and repairing the mitral and tricuspid valves.

The operation was successful. The endotracheal tube was 

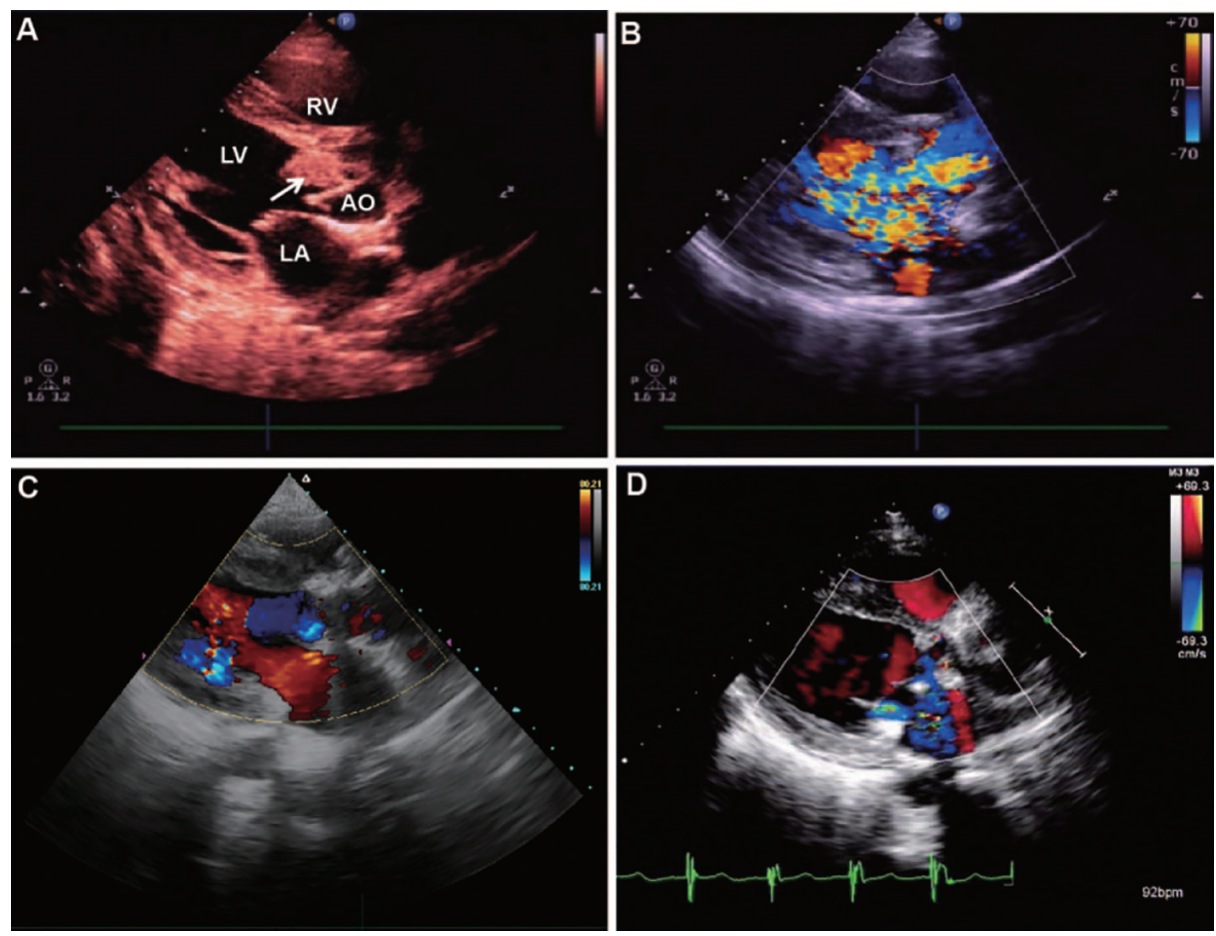

Figure 1. Transthoracic echocardiography demonstrated large vegetation attached at the bicuspid aortic valve leaflet ( $A$, arrow indicated), with mild to moderate aortic stenosis and moderate to severe aortic regurgitation, severe mitral regurgitation, moderate tricuspid regurgitation and dilations of the left atrium and left ventricle (B). The second day after surgery, repeated transthoracic echocardiography demonstrated that valvular regurgitation disappeared (C). Transthoracic echocardiography revealed myocardial contractility similar to normal and no valvular regurgitation still 1 month after the surgery (D). LA: left atrium; LV: left ventricle; RV: right ventricle; $\mathrm{AO}$ : aorta.

removed 2 days after the surgery and the patient had no symptoms of chest tightness or dyspnea. Warfarin, vancomycin, diuretic and inotropic drugs were given. Repeated transthoracic echocardiography demonstrated that the valvular regurgitation disappeared and heart function was slightly better than before (Fig. 1C). Postoperatively, the patient had interrupted fever with highest temperature of $39.0^{\circ} \mathrm{C}$. Red rashes with pruritus were found on the chest and back. White blood count was $8,500 / \mathrm{mm} 3$ with $81.5 \%$ of neutrophils. Considering the adverse event of using vancomycin, the antibiotic was changed to biapenem and teicoplanin, and rashes subsided subsequently. Repeat blood cultures were all negative. Twenty-seven days after cardiac operation, the patient had no fever and biapenem was discontinued. Forty-two days after cardiac operation, the patient was discharged, with no signs or symptoms of clinical heart failure and body temperature was satisfactorily controlled. Transthoracic echocardiography before discharge revealed myocardial contractility similar to normal and no valvular regurgitation still (Fig. 1D). The patient had no fever and dyspnea followed up for 2 months.

\section{Discussion}

The incidence of IE in pregnancy is low and often associated with a previous history of rheumatic or congenital heart disease. It is very hard to select the appropriate management strategy, particularly optimal time and mode of delivery, optimal time and type of valve surgery. According to the current reported cases, delivery was preferred before cardiothoracic surgery or massive antibiotics treatments, in order to save the neonate life as well as to give chance to save the mother $[2,3$, 5]. Here the neonate died by respiratory failure, maybe due to the long time of intrauterine hypoxia. However, we get enough time to save the mother by cardiothoracic surgery and antibiotic drugs.

The indication of surgery for IE patients included uncontrolled fever, refractory heart failure or high risk of systemic embolism by vegetation. In our reported case, the patient had consistent blood temperature of $39.6{ }^{\circ} \mathrm{C}$ after the cesarean, although treated by antibiotics. Meanwhile, she had acute left heart failure, so after 4 days of cesarean, surgical intervention was advocated for the evidence of lack of improvement following conservative managements. At that time, the risk of bleeding was low consulted by both obstetrician and cardiothoracic surgeons. Eventually, the patient recovered and was discharged.

\section{Conclusion}

In conclusion, we treated a case of Staphylococcus aureus in a pregnant woman, by proper aortic valve replacement and antibiotic administration after fetal delivery by cesarean section. 
This is a feasible approach for patients of IE with pregnancy.

\section{Conflicts of Interest}

The authors have no conflicts to disclose.

\section{References}

1. Thanavaro KL, Nixon JV. Endocarditis 2014: an update. Heart Lung. 2014;43(4):334-337.

2. Campuzano K, Roque H, Bolnick A, Leo MV, Campbell
WA. Bacterial endocarditis complicating pregnancy: case report and systematic review of the literature. Arch Gynecol Obstet. 2003;268(4):251-255.

3. Vizzardi E, De Cicco G, Zanini G, D'Aloia A, Faggiano P, Lo Russo R, Chiari E, et al. Infectious endocarditis during pregnancy, problems in the decision-making process: a case report. Cases J. 2009;2:6537.

4. Aoyagi S, Akasu K, Amako M, Yoshikawa K, Hori H. Infective endocarditis during pregnancy: report of a case. Ann Thorac Cardiovasc Surg. 2005;11(1):51-54.

5. Ozer O, Cebesoy FB, Sari I, Davutoglu V. A case of Salmonella typhi endocarditis in pregnancy. Am J Med Sci. 2009;337(3):210-211. 